AN. MED. INTERNA (Madrid) Vol. 19, N. $^{\circ} 6$, pp. 289-295, 2002

\section{Utilidad de la historia clínica, la exploración física y la radiografía en la localización del sangrado de los pacientes con hemoptisis}

\author{
M. HARO ESTARRIOL*, M. VIZCAYA SÁNCHEZ, M. RUBIO GODAY*, \\ J. JIMÉNEZ LÓPEZ, A. NÚÑEZ ARES, A. TORNERO MOLINA \\ Sección de Neumología. Hospital General. Albacete. *Hospital Universitario \\ Doctor Josep Trueta. Girona
}

\begin{abstract}
MEDICAL HISTORY, PHYSICAL EXAMINATION AND CHEST RADIOGRAPHY USEFULLNESS TO LOCALIZE THE SITE OF BLEE DING IN PATIENTS WITH HEMOPTYSIS
\end{abstract}

\title{
RESUMEN
}

Objetivo: Valorar la utilidad de la historia clínica, la exploración física y la radiografía en establecer el origen del sangrado en los pacientes con hemoptisis.

Métodos: Estudio prospectivo, descriptivo y comparativo de 466 casos de hemoptisis para valorar la utilidad de la información obtenida de la historia clínica, la exploración física y la radiografía para localizar el origen del sangrado (derecho o izquierdo) previa confirmación con la broncoscopia, la TC torácica o la arteriografía, considerando de forma especial a las etiologías más frecuentes o el volumen de sangrado.

Resultados: Edad 62,6 años (DE 14), 85\% varones, $80 \%$ fumadores con un sangrado de $42,5 \mathrm{ml} /$ día (DE 86 ) y un volumen $\geq 100 \mathrm{ml} /$ día en el $13,5 \%$. La capacidad de localizar el sangrado aumentó progresivamente con la historia clínica $(1-13 \%, p<0,0001)$, la clínica $(8-29 \%, p<0,0001)$, la exploración física $(13-47,5 \%, \mathrm{p}<0,0001)$ o la radiografía $(14,5-88 \%$, $\mathrm{p}=0,04)$, con unos porcentajes de localización correcta más elevados $(p<0,01)$ y una probabilidad progresivamente mayor de acertar con el mismo orden en la mayoría de grupos, mejorando en las neoplasias y empeorando con las bronquiectasias o bronquitis crónica. En los pacientes con un sangrado $\geq 100 \mathrm{ml} /$ día, la clínica fue más útil en la localización $(\mathrm{p}=0,04)$ que cuando era $<100 \mathrm{ml} /$ día a diferencia de la radiografía $(\mathrm{p}=0,0001)$. La sensibilidad, especificidad y valores predictivos fueron muy variables aunque mejores en la radiografía, disminuyendo con la exploración física, la clínica y la historia clínica.

Conclusiones: Nuestro estudio demuestra la mayor utilidad de la radiografía y la dificultad de la historia clínica, la clínica o la exploración física en localizar el sangrado de los pacientes con hemoptisis. La presencia de hallazgos sugestivos de una localización estuvo prácticamente siempre relacionada con una elección correcta y su probabilidad de acertar aumentó con la radiografía y disminuyó progresivamente con la exploración física, la clínica y especialmente con la historia clínica en los pacientes con bronquiectasias y bronquitis crónica.

PALABRAS CLAVE: Hemoptisis. Localización del sangrado. Radiografía.

Haro Estarriol M, Vizcaya Sánchez M, Rubio Goday M, Jiménez López J, Núñez Ares A, Tornero Molina A. Utilidad de la historia clínica, la exploración física y la radiografía en la localización del sangrado de los pacientes con hemoptisis. An Med Interna (Madrid) 2002; 19: 289-295.

\section{INTRODUCCIÓN}

La hemoptisis puede ser la primera manifestación de un amplio espectro de enfermedades potencialmente graves cuya

\section{ABSTRACT}

Objective: We examined the medical history, physical examination and chest radiography utility to accurately identifying the site of pulmo nary bleeding in patients with hemoptysis.

Methods: We prospectively reviewed and compared the suspected site of bleeding obtained with the medical history, physical examination and chest radiography (right or left) in 466 patients with hemoptysis after the confirmation with a bronchoscopy, computed chest tomography (CT) or bronchial arteriography, and separately analysing the more common etiologies and the volume of bleeding.

Results: Age 62,6 years (DS 14), 85\% males, 80\% smokers with a volume of bleeding of $42,5 \mathrm{ml} /$ day (DS 86) and $\geq 100 \mathrm{ml} /$ day in $13,5 \%$. Medical history localized the site of bleeding in 1-13\% ( $p<0,0001)$, clini cal responses in $8-29 \%(p<0,0001)$, physical examination in $13-47,5 \%$ $(p<0,0001)$ and chest radiography in $14,5-88 \%(p=0,04)$, with a more frequent accurately location findings $(p<0,01)$ that gradually increased as the previous results with the lung carcinomas and decreased with bronchiectasis or chronic bronchitis. When the volume of bleeding was $\geq 100 \mathrm{ml} /$ day, clinical responses utility improved $(p=0,04)$ as when it was $<100 \mathrm{ml} /$ day with the radiography $(p=0,0001)$. Specificity, sensitivity and predictive values were variable and better with the radiogaphy than with the medical history or physical examination.

Conclusions: We concluded that chest radiography was most usefull than the medical history or physical examination to localize the site of bleeding in patients with hemoptysis. Almost all of the findings that sug gests the site of bleeding were accurate and they increased with the radiography or decreased with the physical examination and specially with the medical hystory in patients with bronchiectasis or chronic bron chitis.

KEY WORDS: Hemoptysis. Site of bleeding. Radiography.

Trabajo aceptado: 12 de marzo de 2002

Correspondencia: Dr. Manuel Haro Estarriol. Sección de Neumología (Planta 4ª̈B). Hospital Universitario de Girona "Doctor Josep Trueta”. Avenida de Francia s/n.17007 Girona. e-mail: mip.mharo@htrueta.scs.es

principal preocupación con relación a su manejo está en conocer su etiología, localizar el sangrado e iniciar un tratamiento (1-3). En la mayoría de los pacientes y en función del tipo de sangrado o la etiología, los consensos actuales resaltan la 
importancia de su localización al condicionar el tratamiento por permitir la realización de medidas locales endoscópicas, colocar al paciente en el decúbito lateral del hemitórax patológico para preservar el pulmón sano evitando una asfixia precoz, facilitar la hemostasia por estasis y formación de coágulos, dirigir mejor la búsqueda de la arteria sangrante para embolizarla rápidamente durante la arteriografía o también, para orientar al cirujano en caso de necesitar una toracotomía urgente (1-4).

La broncoscopia, la tomografía computarizada (TC) torácica y la arteriografía son las técnicas más utilizadas para localizar el sangrado en los pacientes con hemoptisis $(1,5)$. No obstante, su rentabilidad es variable y no existe una disponibilidad inmediata o urgente en todos los casos, especialmente en Atención Primaria u hospitales que no son de referencia. En estos casos, el manejo o traslado del paciente con hemoptisis y la localización del sangrado deben basarse en la experiencia del médico al recurrir a la historia clínica, la exploración física o la radiografía, como únicas exploraciones inicialmente disponibles aunque habitualmente infravaloradas, de una rentabilidad poco conocida y sin una importancia bien establecida en todos los casos $(1,2,4,6)$. En estas circunstancias, hemos planteado como objetivo de nuestro estudio el conocimiento de la utilidad de la historia clínica, la exploración física y la radiografía simple de tórax en la localización del sangrado de los pacientes con hemoptisis, prestando especial atención a las etiologías más frecuentes y al volumen del sangrado.

\section{MÉTODOS}

Estudio prospectivo y descriptivo de los pacientes valorados por una hemoptisis en el periodo comprendido entre Enero de 1996 y Diciembre de 1999, incluyendo todos aquellos casos remitidos a una consulta monográfica o ingresados en un hospital general de unas 600 camas y un área de referencia de unos 395.000 habitantes; considerando como hemoptisis a la expulsión de sangre por la boca mediante la tos y de una procedencia traqueobronquial excluyendo aquellos pacientes cuyo sangrado fuera digestivo o nasofaríngeo.

Una vez establecida la presencia de una hemoptisis se determinó la edad, sexo, tabaquismo, volumen diario, duración y la etiología del sangrado según un protocolo realizado en nuestro centro $(7,8)$. Con la intención de localizar el origen de la hemoptisis (derecho o izquierdo) se evaluó de forma independiente la información obtenida de: a) una historia clínica intencionada con relación a sangrados previos y su localización; b) los signos o síntomas que para el paciente eran sugestivos de un sangrado en un determinado hemitórax; c) una auscultación pulmonar que demostrara anomalías locales o asimétricas que permitieran sospechar un posible origen del sangrado; y d) la radiografía simple de tórax analizando la existencia de lesiones potencialmente sangrantes en las proyecciones posteroanterior y de perfil.

Para establecer una referencia de la localización del sangrado con relación al resto de las exploraciones analizadas hemos utilizado la broncoscopia (FB) y/o la TC torácica, junto a la arteriografía bronquial en aquellos pacientes en que fue realizada. Los pacientes fueron sometidos a una FB para valorar el árbol traqueobronquial empleando como instrumental un fibrobroncoscopio Olympus modelo OES10 y OES P2OD (Medical Europa S.A.), con la excepción de los casos con una sospecha y/o confirmación etiológica que la hacía innecesaria o contraindicaba (tromboembolismo pulmonar, aneurisma de aorta,...). La FB estableció la localización del sangrado cuando se detectaron lesiones endobronquiales potencialmente sangrantes (tumoraciones, lesiones sospechosas de una neoplasia o un cuerpo extraño), sangrado activo o restos hemáticos de aspecto reciente aislados en determinadas zonas de un único segmento, lóbulo o hemitórax. La TC fue realizada sin conocer los resultados de la FB a todos aquellos pacientes que cumplían alguno de los siguientes criterios: a) sospecha de una neoplasia pulmonar; b) sospecha clínica o radiológica de bronquiectasias; c) una hemoptisis recidivante (episodios repetidos en un tiempo de 1-2 meses); d) persistencia de un sangrado superior a los 10 días sin un diagnóstico claro o que lo justificara; e) un volumen de sangrado superior a $50 \mathrm{ml} / \mathrm{día}$; y f) para valorar anomalías poco definidas en la radiografía simple o en otras etiologías en las que pudiera ser de ayuda. Esta exploración fue realizada administrando contraste intravenoso con cortes de 1-2 mm cada $10 \mathrm{~mm}$ desde el estrecho torácico superior hasta las bases pulmonares, disminuyendo el intervalo a $5 \mathrm{~mm}$ en las bases o zonas sospechosas de alguna patología. Los resultados fueron evaluados independientemente por un radiólogo y un neumólogo, cuyo acuerdo permitió clasificar a la TC como normal o con alteraciones específicas relacionadas con la hemoptisis (sugestivas o sospechosas de un sangrado reciente o de una etiología potencialmente sangrante) y su localización.

Se realizó un análisis descriptivo de la utilidad de la historia clínica (anamnesis y clínica), la exploración física y la radiografía para localizar el sangrado, junto a un estudio comparativo de dicha utilidad en todos los casos, las etiologías más frecuentes o según el volumen del sangrado mediante la t de Student para variables cuantitativas y la ji cuadrado o el test exacto de Fisher cuando fue necesario para las variables cualitativas considerando un resultado estadísticamente significativo cuando la p era inferior a 0,05 . Se ha analizado la sensibilidad (S), especifidad (E), valor predictivo positivo (VPP) y negativo (VPN) para cada una de las exploraciones y grupos de estudio. Se han calculado los cocientes de probabilidad o verosimilitud (CP o likelihood ratio) para analizar la magnitud o dirección de la capacidad de localizar acertadamente el lugar del sangrado de las distintas exploraciones, considerando que solo los valores por encima de uno la aumentaban.

\section{RESULTADOS}

En los 752 pacientes consecutivos con hemoptisis estudiados inicialmente se realizaron un total de $626 \mathrm{FB}(83,2 \%)$, 516 TC torácicas $(79,4 \%)$ y 39 arteriografías bronquiales $(5,6 \%)$ para su valoración. La etiología de los pacientes estudiados queda reflejada en la tabla I. De estos casos fueron excluidos $286(38 \%)$, divididos en 17 pacientes donde la FB o la arteriografía objetivaron un sangrado activo bilateral (6\%), 35 casos en que la combinación de la FB y la TC no fueron capaces de localizar el sangrado (12\%), 45 en que la FB no fue definitiva y la TC objetivó lesiones bilaterales potencialmente sangrantes (16\%), 106 en los que se realizó solo una TC o una FB que no permitieron localizar el sangrado (37\%) y finalmente, 83 casos (29\%) fueron excluidos al no realizarse una TC o una FB por la negación o falta de colaboración del paciente en el caso de la FB y por la escasa cuantía o una 
TABLA I

DISTRIBUCIÓ N ETIO LÓ GICA DE TODOS LOS CASOS DE HEM O PTISIS, LOS ESTUDIADOS Y LOS QUE PRESENTABAN UN VOLUM EN DE SANGRADO SUPERIOR A $100 \mathrm{ml}$ / DÍA

\begin{tabular}{|c|c|c|c|c|c|}
\hline & $\begin{array}{l}\text { Casos } \\
\text { Totales }\end{array}$ & $\begin{array}{c}\text { Casos } \\
\text { Estudio }\end{array}$ & $\%$ & $\begin{array}{l}\text { Casos } \\
\geq 100 \\
\mathrm{ml} / \text { día }\end{array}$ & $\%$ \\
\hline Neoplasias & 210 & 200 & 43 & 6 & 9,5 \\
\hline $\begin{array}{l}\text { Carcinoma epidermoide } \\
\text { CPCP } \\
\text { Adenocarcinoma } \\
\text { Carcinoma indiferenciado } \\
\text { M etástasis } \\
\text { Neoplasia esófago } \\
\text { Carcinoma células grandes } \\
\text { Tumor carcinoide }\end{array}$ & $\begin{array}{c}109 \\
42 \\
25 \\
12 \\
9 \\
4 \\
3 \\
3\end{array}$ & & & & \\
\hline M iscelánea & 3 & & & & \\
\hline Bronquitis crónica & 149 & 62 & 13,3 & 14 & 22,2 \\
\hline Bronquiectasias & 109 & 80 & 17,1 & 22 & 35 \\
\hline Neumonía/Absceso & 86 & 51 & 11 & 3 & 4,8 \\
\hline Cicatrices extuberculosas & 29 & 21 & 4,5 & 6 & 9,5 \\
\hline Asma & 26 & 3 & 0,6 & 0 & 0 \\
\hline TEP & 17 & 3 & 0,6 & 0 & 0 \\
\hline Tuberculosis pulmonar & 13 & 6 & 1,3 & 2 & 3,2 \\
\hline Cardiopatía/Valvulopatía & 11 & 1 & 0,2 & 0 & 0 \\
\hline Aspergiloma & 7 & 7 & 1,5 & 4 & 6,3 \\
\hline Cuerpo extraño & 4 & 4 & 0,9 & 0 & 0 \\
\hline M iscelánea & 30 & 15 & 3,2 & 5 & 7,9 \\
\hline Criptogenéticas & 61 & 13 & 2,8 & 1 & 1,6 \\
\hline TOTAL & 752 & 466 & 100 & 63 & 100 \\
\hline
\end{tabular}

CPCP Carcinoma pulmonar de células pequeñas. TEP Tromboembolismo pulmonar.

duración del sangrado inferior a 3 días en enfermos cuyos diagnósticos fueron confirmados tras su seguimiento y la ausencia de nuevos sangrados. Los 466 casos restantes (62\%) fueron motivo del estudio al localizar un sangrado exclusivamente en un único lóbulo, segmento o hemitórax. Las características de estos pacientes incluían una edad de 62,6 años (DE desviación estándar 14, rangos 17-93 años y una edad inferior a 45 años en un 18\%), 396 varones (85\%) y 374 fumadores (80\%) de 52 paquetes/año (DE 32). El sangrado fue de 42,5 $\mathrm{ml} /$ día (DE 86 y rangos 5-600 ml/día), con un volumen igual o superior a $100 \mathrm{ml} /$ día en 63 pacientes $(13,5 \%)$ y una duración de 14 días (DE 24 y rangos de 1-180 días). La localización de referencia u origen del sangrado en este subgrupo se realizó con la FB en 395 de las 455 exploraciones realizadas en estos pacientes $(87 \%, 50$ sangrados activos, 181 con restos hemáticos localizados y 164 por sospecha de neoplasia), complementada por la TC torácica en 375 de las 417 exploraciones realizadas $(91 \%)$. En este subgrupo se realizaron 31 arteriografías bronquiales con intención terapéutica que confirmaron el posible origen del sangrado y lesiones potencialmente sangrantes no activas contralaterales en 7 casos $(22,6 \%)$. La tabla I muestra la distribución de las etiologías de los pacientes estudiados. La miscelánea de la etiología de las neoplasias del grupo de estudio incluía casos únicos de un tumor glómico, un carcinoma adenoide quístico y un papiloma endobronquial. La miscelánea de las etiologías no neoplásicas incluyó a seis casos de contusión pulmonar, dos neumonías lipoideas, dos lupus eritematosos con afectación pulmonar, tres síndromes de Goodpasture y casos únicos de granulomatosis de Wegener o bronquiolitis obliterante con neumonía organizada.

Las tablas II y III reflejan los resultados de las distintas exploraciones utilizadas para localizar el sangrado. Ambas tablas muestran su rentabilidad en localizarlo de forma global, según las etiologías más frecuentes y el volumen de sangrado, diferenciando los motivos y si coincidía o no con las técnicas de referencia para cada grupo (correcta o incorrecta). En la clínica, los pacientes localizaron el origen del sangrado por la presencia de dolor torácico, la audición de ruidos anormales en forma de "gorgoteo" o por la presencia de molestias torácicas inespecíficas ausentes antes del sangrado. En la exploración física, por la presencia asimétrica y localizada en la auscultación de hipofonesis, roncus o sibilantes y estertores, mientras en la radiografía se consideraron significativas la presencia de una condensación o infiltrado pulmonar, una atelectasia, una masa o nódulo pulmonar, un hilio pulmonar anormal, un absceso o cavitación y lesiones pulmonares loca- 
TABLA II

RESULTADOS Y PORCENTAJES DE LA UTILIDAD DE LA HISTO RIA CLÍNICA

Y LA CLIINICA EN LA LOCALIZACIÓN DEL SANGRADO

\begin{tabular}{lcccccc}
\hline & $\begin{array}{c}\text { Global } \\
\text { Totales }\end{array}$ & Neoplasia & $\begin{array}{c}\text { Bronquitis } \\
\text { Crónica }\end{array}$ & BQ & $\begin{array}{c}\text { Sangrado } \\
<100 \\
\mathrm{ml} / \text { día }\end{array}$ & $\begin{array}{c}\text { Sangrado } \\
\geq 100 \\
\mathrm{ml} / \text { día }\end{array}$ \\
\hline $\mathrm{N}$ & 466 & 200 & 62 & 80 & 403 & 63 \\
Historia Clínica & & & & & & \\
$\quad$ Hemoptisis previa (\%) & $124(27)$ & $26(13) \mathrm{b}$ & $24(38,7)$ & $42(52,5)$ & $98(24,5) \mathrm{c}$ & $26(41)$ \\
Localización (\%) & $35(7)$ & $3(1) \mathrm{b}$ & $9(14)$ & $19(23)$ & $27(6,7)$ & $8(12,7)$ \\
Correcta (\%) & $23(66) \mathrm{a}$ & $2(66,6) \mathrm{a}$ & $5(55,5)$ & $12(63)$ & $18(66,6) \mathrm{a}$ & $5(62,5)$ \\
Incorrecta (\%) & $12(44)$ & $1(33,3)$ & $4(45,5)$ & $7(37)$ & $9(33,3)$ & $3(37,5)$ \\
Clínica & & & & & & \\
Localización (\%) & $87(19)$ & $37(18,5)$ & $5(8,1)$ & $16(20)$ & $69(17) \mathrm{c}$ & $18(28,6)$ \\
Correcta (\%) & $80(92) \mathrm{a}$ & $37(100) \mathrm{a}, \mathrm{b}$ & $3(60) \mathrm{a}$ & $11(69) \mathrm{a}$ & $63(91) \mathrm{a}$ & $17(94,4) \mathrm{a}$ \\
$\quad$ Dolor & 58 & 31 & 2 & 4 & 56 & 2 \\
$\quad$ Ruidos anómalos & 20 & 6 & 1 & 6 & 6 & 14 \\
$\quad$ Molestias inespecíficas & 2 & 0 & 0 & 1 & 1 & 1 \\
Incorrecta (\%) & $7(8)$ & $0 \mathrm{~b}$ & $2(40)$ & $5(31)$ & $6(9)$ & $1(5,6)$ \\
$\quad$ Dolor & 4 & 0 & 1 & 3 & 3 & 1 \\
$\quad$ Ruidos anómalos & 3 & 0 & 1 & 2 & 3 & 0 \\
\hline
\end{tabular}

BQ Bronquiectasias. ap <0,05 en la relación correcta e incorrecta de una misma prueba y grupo. bp $<0,05$ en relación entre los tres grupos de etiologías más frecuentes. $\mathrm{cp}<0,05$ entre los grupos establecidos por el volumen de sangrado ( $<100 \mathrm{ml} / \mathrm{día} 0 \geq 100 \mathrm{ml} / \mathrm{día}$ ).

lizadas de aspecto cicatricial. La capacidad de localizar el sangrado en el análisis global siempre fue inferior en la historia clínica ( $p<0,0001)$, seguido de la clínica $(p<0,0001)$, la exploración física $(\mathrm{p}<0,0001)$ y la radiografía como la más rentable $(\mathrm{p}=0,04)$, de forma similar al resto de grupos del estudio a excepción de los pacientes con bronquitis crónica o bronquiectasias con una capacidad de localización similar en todas las exploraciones menos en la radiografía donde se incrementaba en el grupo de las bronquiectasias $(\mathrm{p}=0,03)$. En todos los grupos comparados y las distintas exploraciones, las respuestas correctas superaron a las incorrectas $(\mathrm{p}<0,01)$ con la excepción de la historia clínica en los pacientes con bronquitis crónica y bronquiectasias. En los grupos realizados a partir del volumen de sangrado, los resultados fueron similares en el mayor número de respuestas correctas y se diferenciaron por una mayor utilidad en localizar el sangrado de la clínica cuando era $\geq 100 \mathrm{ml} /$ día $(\mathrm{p}=0,04)$ y de la radiografía en los que era inferior $(\mathrm{p}=0,0001)$. Entre los grupos de las etiologías más frecuentes destacó la menor utilidad de la historia clínica $(\mathrm{p}<0,0001)$ y una mayor capacidad de la exploración física $(p=0,003)$ o la radiografía $(p=0,0001)$ en localizar el sangrado en los pacientes con una neoplasia, así como un mayor número de aciertos en localizarlo de forma correcta en todas las exploraciones analizadas de este mismo grupo a excepción de la historia clínica ( $\mathrm{p}=0,01$ en la clínica, $\mathrm{p}=0,002$ en la exploración física y $\mathrm{p}=0,04$ en la radiografía).

La tabla IV presenta los valores de los cocientes de probabilidad para acertar una localización a partir de las distintas exploraciones y los mismos grupos. La tabla V muestra la especificidad, sensibilidad, valor predictivo positivo y negati- vo de las exploraciones realizadas en este estudio para la valoración general, de las etiologías más frecuentes y con relación al volumen del sangrado.

\section{DISCUSIÓN}

Nuestro estudio demuestra la mayor utilidad de la radiografía y la dificultad de la historia clínica, la clínica o la exploración física en localizar el sangrado en este grupo de pacientes con hemoptisis en relación a otras exploraciones utilizadas habitualmente como referencia $(9,11)$. Esta dificultad aumenta especialmente con la historia clínica y mejora o disminuye de forma progresiva si utilizamos la información obtenida de la clínica, la exploración física y la radiografía, esta última alcanzando un porcentaje del $69 \%$ en la valoración conjunta de todos los casos o del $88 \%$ de las neoplasias. No obstante, considerando su fácil realización o disponibilidad en la mayoría de centros que valoran inicialmente estos pacientes, la presencia de los hallazgos que hemos establecido como significativos para cada exploración estuvo prácticamente siempre relacionada con una correcta localización con la excepción de la historia clínica, y su capacidad o probabilidad de acertar aumentó progresivamente con el mismo orden, llegando a los valores más favorables con la radiografía y en las neoplasias (Tabla IV y V).

La historia clínica o la disponibilidad de un antecedente que sugiriera el origen del sangrado fue la determinación menos rentable y con una menor probabilidad o mayor dificultad de acertar, especialmente en los pacientes con bronqui- 


\section{TABLA III}

RESULTADOSY PORCENTAJES DE LA UTILIDAD DE LA EXPLORACIÓN FÍSICA Y LA RADIO GRAFÍA EN LA LOCALIZACIÓN DEL SANGRADO

\begin{tabular}{|c|c|c|c|c|c|c|}
\hline & $\begin{array}{l}\text { Global } \\
\text { Totales }\end{array}$ & Neoplasia & $\begin{array}{l}\text { Bronquitis } \\
\text { Crónica }\end{array}$ & $B Q$ & $\begin{array}{c}\text { Sangrado } \\
<100 \\
\mathrm{ml} / \mathrm{d} i ́ \mathrm{a}\end{array}$ & $\begin{array}{c}\text { Sangrado } \\
\geq 100 \\
\mathrm{ml} / \mathrm{d} \text { día }\end{array}$ \\
\hline $\mathrm{N}$ & 466 & 200 & 62 & 80 & 403 & 63 \\
\hline \multicolumn{7}{|l|}{ Exploración Física } \\
\hline Localización (\%) & $184(39,5)$ & $95(47,5)$ & $8(13)^{b}$ & $29(36)$ & $157(39)$ & $27(43)$ \\
\hline Correcta $(\%)$ & $177(96,2)^{\mathrm{a}}$ & $95(100)^{a, b}$ & $6(75)$ & $25(86)^{a}$ & $154(98)^{a, c}$ & $23(85)^{a}$ \\
\hline Hipofonesis & 96 & 72 & 1 & 3 & 87 & 9 \\
\hline Roncus/ Sibilancias & 25 & 15 & 1 & 6 & 23 & 2 \\
\hline Estertores & 56 & 8 & 4 & 16 & 44 & 12 \\
\hline Incorrecta (\%) & $7(3,8)$ & $0^{\mathrm{b}}$ & $2(25)$ & $4(14)$ & $3(2) c$ & $4(15)$ \\
\hline Hipofonesis & 3 & 0 & 0 & 0 & 1 & 2 \\
\hline Broncoespasmo & 1 & 0 & 1 & 1 & 1 & 0 \\
\hline Estertores & 3 & 0 & 1 & 3 & 1 & 2 \\
\hline \multicolumn{7}{|l|}{ Radiografía } \\
\hline Localización (\%) & $321(69)$ & $176(88)^{b}$ & $9(14,5)^{b}$ & $41(51,3)^{\mathrm{b}}$ & $291(72)^{c}$ & $30(48)$ \\
\hline Correcta (\%) & $316(98,4)^{\mathrm{a}}$ & $176(100)^{a, b}$ & $8(89)^{a}$ & $39(95)^{\mathrm{a}}$ & $288(99)^{\mathrm{a}}$ & $28(93,3)^{a}$ \\
\hline Condensa/infiltrado & 120 & 34 & 7 & 31 & 109 & 11 \\
\hline Atelectasia & 48 & 40 & 1 & 2 & 43 & 5 \\
\hline M asa/Nódulo & 68 & 64 & 0 & 0 & 67 & 1 \\
\hline Hilio anormal & 25 & 26 & 0 & 0 & 20 & 5 \\
\hline Absceso/ cavitación & 29 & 7 & 0 & 0 & 26 & 3 \\
\hline Cicatrices localizadas & 26 & 5 & 0 & 6 & 23 & 3 \\
\hline Incorrecta (\%) & $5(1,6)$ & $0^{\mathrm{b}}$ & $1(11)^{b}$ & $2(5)^{b}$ & $3(1)$ & $2(6,7)$ \\
\hline Condensa/ infiltrado & 3 & 0 & 1 & 2 & 2 & 1 \\
\hline Atelectasia & 1 & 0 & 0 & 0 & 1 & 0 \\
\hline Hilio anormal & 0 & 0 & 0 & 0 & 0 & 0 \\
\hline Cicatrices localizadas & 1 & 0 & 0 & 0 & 0 & 1 \\
\hline
\end{tabular}

TABLA IV

COCIENTES DE PRO BABILIDAD O VEROSIMILITUD (CP) DE ACERTAR LA LOCALIZACIÓN DEL SANGRADO DE LAS DISTINTAS EXPLO RACIO NES ESTUDIADAS

\begin{tabular}{|c|c|c|c|c|c|c|}
\hline & $\begin{array}{l}\text { Global } \\
\text { Totales }\end{array}$ & Neoplasia & $\begin{array}{c}\text { Bronquitis } \\
\text { Crónica }\end{array}$ & $\mathrm{BQ}$ & $\begin{array}{c}\text { Sangrado } \\
<100 \\
\mathrm{ml} / \mathrm{día}\end{array}$ & $\begin{array}{c}\text { Sangrado } \\
\geq 100 \\
\mathrm{ml} / \mathrm{d} \text { día }\end{array}$ \\
\hline $\begin{array}{r}\text { Historia clínica } \\
\text { Localización }\end{array}$ & 1,5 & 2 & 1,2 & 1,7 & 2 & 1,7 \\
\hline $\begin{array}{l}\text { Clínica } \\
\quad \text { Localización }\end{array}$ & 11,5 & 36 & 1,5 & 2,2 & 10,1 & 15,6 \\
\hline $\begin{array}{l}\text { Exploración física } \\
\text { Localización }\end{array}$ & 25,3 & 25,2 & 3 & 6,6 & 49 & 6,1 \\
\hline $\begin{array}{l}\text { Radiografía } \\
\text { Localización }\end{array}$ & 61,3 & 198 & 8 & 19 & 99 & 15,5 \\
\hline
\end{tabular}

BQ Bronquiectasias. 


\section{TABLA V}

RESULTADOS DE LA SENSIBILIDAD (S), ESPECIFICIDAD (E), VALOR PREDICTIVO PO SITIVO (VPP) Y NEGATIVO (VPN) DE LAS EXPLO RACIO NES REALIZADAS DE FO RM A GLO BAL, SEGÚN LAS ETIO LO GÍAS M ÁS FRECUENTES $Y$ EL VOLUM EN DE SANGRADO

\begin{tabular}{|c|c|c|c|c|}
\hline & $S(\%)$ & $E(\%)$ & $\operatorname{VPP}(\%)$ & VPN $(\%)$ \\
\hline \multicolumn{5}{|c|}{$\begin{array}{l}\text { Global } \\
\text { Neoplasia } \\
\text { Bronquitis Crónica } \\
\text { Bronquiectasias } \\
\text { Sangrado <100 ml/ día } \\
\text { Sangrado } \geq 100 \mathrm{ml} / \text { día }\end{array}$} \\
\hline Historia Clínica & $\begin{array}{l}66 \\
66 \\
55 \\
63 \\
66 \\
62\end{array}$ & $\begin{array}{l}77 \\
88 \\
72 \\
62 \\
81 \\
67\end{array}$ & $\begin{array}{c}19 \\
8 \\
25 \\
34 \\
20 \\
21\end{array}$ & $\begin{array}{l}96 \\
99 \\
90 \\
84 \\
97 \\
92\end{array}$ \\
\hline Clínica & $\begin{array}{c}91 \\
100 \\
60 \\
68 \\
91 \\
94\end{array}$ & $\begin{array}{l}89 \\
89 \\
89 \\
89 \\
90 \\
91\end{array}$ & $\begin{array}{l}68 \\
67 \\
33 \\
69 \\
65 \\
81\end{array}$ & $\begin{array}{c}98 \\
100 \\
96 \\
92 \\
98 \\
98\end{array}$ \\
\hline Exploración Física & $\begin{array}{c}96 \\
100 \\
75 \\
86 \\
98 \\
85\end{array}$ & $\begin{array}{c}46 \\
47 \\
61 \\
41 \\
49 \\
100\end{array}$ & $\begin{array}{c}57 \\
63 \\
28 \\
45 \\
55 \\
100\end{array}$ & $\begin{array}{c}83 \\
100 \\
94 \\
84 \\
97 \\
90\end{array}$ \\
\hline Radiografía & $\begin{array}{c}98 \\
100 \\
88 \\
95 \\
98 \\
93\end{array}$ & $\begin{array}{c}62 \\
100 \\
86 \\
94 \\
89 \\
88\end{array}$ & $\begin{array}{c}85 \\
100 \\
53 \\
95 \\
96 \\
88\end{array}$ & $\begin{array}{c}95 \\
100 \\
98 \\
95 \\
97 \\
93\end{array}$ \\
\hline
\end{tabular}

tis crónica y bronquiectasias, a pesar de ser los que más episodios previos de hemoptisis referían y donde se esperaba una mayor utilidad por la posibilidad de que fuera recidivante. Aunque la sensibilidad o especificidad fueran aceptables, unos resultados muy poco favorables del valor predictivo positivo no permitieron recomendarla como único parámetro de decisión sobre el origen del sangrado cuando era sugerido en este tipo de pacientes.

Los hallazgos de la clínica solo mejoraron parcialmente los obtenidos por la historia clínica y fueron claramente inferiores a los de la exploración física o la radiografía. Su inespecificidad y detección en un número limitado de pacientes contrastó con su utilidad en localizar el sangrado en el análisis global o las neoplasias a diferencia de otros estudios como el de Pursel y cols. (12), donde solo el $10 \%$ de 105 pacientes localizaron el sangrado por la clínica y en el $30 \%$ de forma errónea. La mejora de los valores de la sensibilidad, la especi- ficidad o el valor predictivo positivo y una probabilidad superior de acertar correctamente aconsejaron su búsqueda intencionada o su consideración en estos pacientes, sobre todo si existía un mayor volumen de sangrado. En estos últimos y considerando su mayor riesgo, la sospecha del origen del sangrado en casi el 30\% de los casos, un aumento del cociente de verosimilitud y una mayor probabilidad de acertar con una mayor sensibilidad, especificidad y valor predictivo positivo establecieron su importancia y recomendaron su determinación rutinaria.

La utilidad de la exploración física solo fue superada por la radiografía. Su simplicidad y la posibilidad de realizarla de forma repetida sin riesgos para el paciente en busca de la asimetría de unos hallazgos habitualmente inespecíficos permitió localizar el sangrado en el $30-50 \%$ de los casos con un porcentaje y una probabilidad de aciertos más elevado a excepción de los casos diagnosticados de bronquitis crónica, en los 
que el carácter difuso o bilateral de su enfermedad y la mayor distribución del sangrado con la tos podría ser la justificación de forma similar a descripciones previas en series retrospectivas con un menor número de casos $(2,12)$.

La radiografía de tórax fue la exploración más útil para localizar el sangrado en nuestro estudio. A pesar de que suele ser considerada como una técnica insuficiente para orientar la etiología o localizar el origen del sangrado en la mayoría de los pacientes (13-16), su mayor disponibilidad la convierte en una de las primeras técnicas realizadas y los resultados actuales no permiten despreciarla para localizar el sangrado antes o cuando no tengamos posibilidad de realizar una TC, una arteriografía o una FB de forma urgente (17). La presencia de distintos patrones fácilmente identificables permitió establecer el origen del sangrado en una gran mayoría de los casos con un porcentaje de aciertos casi completo y los cocientes de probabilidad más altos de todas las exploraciones, con la excepción de los pacientes con un mayor sangrado, bronquiectasias y especialmente los diagnosticados de una bronquitis crónica. En los dos últimos podría atribuirse a que estas enfermedades se caracterizan por una afectación más difusa o bilateral y una mayor inespecificidad de sus hallazgos en esta exploración, donde en la mayoría de las series constituyen la mayor parte de los casos con una radiografía normal o inespecífica y obligan a utilizar otras técnicas para conocer el origen del sangrado $(8,16,18$ $20)$. En los pacientes con un mayor sangrado, la menor utilidad de la radiografía podría estar con relación a las etiologías que lo provocaron, coincidiendo mayoritariamente con los mismos diagnósticos de bronquitis crónica o bronquiectasias (63 casos, $57 \%$ ) y la baja prevalencia de neoplasias, neumonías, abscesos pulmonares u otras lesiones con una radiografía más característica (16), aunque la FB o la TC tampoco van a ser infalibles en todos los casos (21-25). No obstante, la sensibilidad, la especificidad y los valores predictivos de nuestros hallazgos en este grupo de pacientes demuestran que la radiografía fue de gran ayuda para detectar el origen del sangrado. Aunque habitualmente solo son considerados como simples estimaciones de la utilidad de las pruebas utilizadas, la aparición de unos mayores valores predictivos confirmó que la presencia de dichos hallazgos contribuía a asegurar con mucha probabilidad la localización del sangrado y su ausencia al desconocimiento del mismo, todo ello complementado por los valores del CP, cuyo resultado confirma la mayor probabilidad de acertar cuando el sangrado es unilateral y los pacientes son de características similares a los nuestros.

\section{AGRADECIMIENTOS}

Este trabajo ha podido realizarse gracias a la colaboración desinteresada de Carmen Blasco, $\mathbf{M}^{\mathrm{a}}$ Jesús García, $\mathbf{M}^{\mathrm{a}}$ José García, Concepción Lázaro, Mercedes Martínez, Juana Tendero, Masosé Andicoberri y el resto del equipo de enfermería de nuestra Sección.

\section{Bibliografía}

1. Roig J, Llorente JL, Ortega FJ, Orriols R, Segarra A (Grupo de Trabajo de la SEPAR). Normativa sobre el manejo de la hemoptisis amenazante. Arch Bronconeumol 1997; 33: 31-40.

2. Cahill BC, Ingbar DH. Massive hemoptysis. Clin Chest Med 1994; 15: 147-68.

3. Colice GL. Hemoptysis. Three questions that can direct management. Postgraduate Med 1996; 100: 227-34

4. Israel RH, Poe RH. Hemoptysis. Clin Chest Med 1987; 8: 197-205.

5. Saumench J, Escarrabill J, Padro L, Montana J, Clariana A, Cantó A. Value of fiberoptic bronchoscopy and angiography for diagnosis of the bleeding site in hemoptysis. Ann Thorac Surg 1989; 48: 272-4.

6. Hirshberg B, Biran Y, Glazer M, Kramer MR. Hemoptysis: etiology, evaluation and outcome in a tertiary referral hospital. Chest 1997; 112: 440-4.

7. Haro M, Núñez A, Vizcaya M, Tirado R, Fernández JM, Muñoz F. Importancia del sexo e influencia del tabaco en la etiología de los pacientes con hemoptisis. An Med Interna (Madrid) 1999; 16: 23943

8. Haro M, Vizcaya M, Jiménez J, Tornero A. Etiología de la hemoptisis. Análisis prospectivo de 752 casos. Rev Clin Esp 2001; 201: 696-700.

9. Martin C, Romero S, Aparicio JM. Utilidad de la fibrobroncoscopia en los pacientes con hemoptisis y una radiografía normal. Rev Clin Esp 1990; 186: 259-63.

10. Müller NL. Hemoptysis: High-resolution CT vs bronchoscopy. Chest 1994; 105: 982-3.

11. Haponik EF, Britt EJ, Smith PL, Bleecker ER. Computed chest tomography in the evaluation of hemoptysis. Impact on diagnosis and treatment. Chest 1987; 91: 80-5.

12. Pursel SE, Lindskog GE. Hemoptysis: a clinical evaluation of 105 patients examined consecutely on a thoracic surgical service. Am Rev Respir Dis 1961; 84: 329-36.

13. Millar AB, Boothroyd AE, Edwards D, Hetzel MR. The role of computed tomography (CT) in the investigation of unexplained hemoptysis.

Respir Med 1992; 86: 39-44.

14. McGuiness G, Beacher JR, Harkin TJ, Garay SM, Rom WN, Naidich DP. Hemoptysis: prospective high-resolution CT/bronchoscopic correlation. Chest 1994; 105: 1155-62.

15. Set PAK, Flower CDR, Smith IE, Chan AP, Twentyman OP, Shneerson JM. Hemoptysis: comparative study of the role of CT and fiberoptic bronchoscopy. Radiology 1993; 189: 677-80.

16. Tasker AD, Flower CDR. Imaging the airways. Hemoptysis, bronchiectasis and small airways disease. Clin Chest Med 1999; 20: 761-73.

17. Pedrol E, Fernández J, Ferrer M, Barcelo J, Bosch X, Sande L et al. Hemoptisis: estudio prospectivo de 108 casos en un servicio de urgencias. Rev Clin Esp 1991; 188: 450-4.

18. Plaza V, Serra J, Falcó M, Brugués J. ¿Han variado las causas de hemoptisis?. Análisis de 213 casos sometidos a exploración fibrobroncoscópica. Arch Bronconeumol 1995; 31: 323-7.

19. Romero S, Martín C, Hernández L. Pronóstico a corto plazo de las hemoptisis criptogenéticas. Rev Clin Esp 1989; 185: 184-6.

20. Adelman M, Haponik EF, Bleecker ER, Britt EJ. Crytogenic hemoptysis. Clinical features, bronchoscopic findings and natural history in 67 patients. An Intern Med 1985; 102: 829-34.

21. Arney KL, Judson MA, Sahn SA. Airway obstruction arising from blood clot. Chest 1999; 115: 293-300.

22. Eisenhuber E, Brunner C, Bankier A. Blood clots mimicking peripheral intrabronchial tumors in patients with hemoptysis: CT and bronchoscopic findings. J Comput Assist Tomogr 2000; 24: 47-51.

23. Naidich DP, Harkin TJ. Airways and lung: correlation of CT with fiberoptic bronchoscopy. Radiology 1995; 197: 1-12.

24. Agustí C, Xaubet A, Torres A. Diagnóstico de la hemoptisis: ¿realmente existe polémica?. An Med Interna (Madrid) 2002; 19: 57-8.

25. Haro M, Jiménez J, Tornero A, Vizcaya M, Tirado R, Cros T. Utilidad de la tomografía computarizada y la broncoscopia en los pacientes con hemoptisis. Análisis de 482 casos. An Med Interna (Madrid) 2002; 19: $59-65$. 